\title{
Le taux d'actualisation en assurance
}

\author{
par Pierre Devolder*
}

\section{Introduction}

Le taux d'actualisation joue un rôle déterminant dans la vie d'une compagnie d'assurance.

En effet, d'une part, les assureurs sont des investisseurs institutionnels importants et intègrent à ce titre dans leur politique de placement des hypothèses quant au taux d'actualisation, au même titre que tout autre investisseur. D'autre part, les produits proprement dits vendus par les assureurs-vie impliquent un rôle central pour les taux d'intérêt.

Nous nous concentrons principalement sur cet aspect spécifique au monde de l'assurance.

\section{A. La technique de l'assurance-vie et le taux d'actualisation}

\section{Généralités}

L'actualisation constitue l'outil central de calcul des primes et des réserves des contrats d'assurance-vie.

En effet, un contrat d'assurance-víe oblige l'assureur, contre versement par l'assuré de primes fixées à l'origine, à servir à celui-ci une prestation déterminée en cas de survenance dans l'avenir d'un événement aléatoire bien défini.

Ce peut être une opération «vie» consistant à verser un capital fixé au terme du contrat si l'assuré est en vie à cette date, ou une opération «décès» consistant à verser un capital fixé au décès de l'assuré si ce décès se produit avant le terme du contrat, ou enfin une combinaison de ces deux opérations.

Dans chaque cas, l'assuré s'engage à verser, par exemple chaque année, une prime tant qu'il est en vie.

L'assureur utilise une partie de ces primes pour le risque, le solde alimentant pour chaque contrat une réserve dite mathématique.

* Royale Belge, Bruxelles 


\section{Le calcul des primes}

Un contrat d'assurance-vie comporte, d'une part, des engagements de la part de l'assureur (versement d'un capital), d'autre part, des engagements de la part de l'assuré (paiement des primes). Ces engagements sont répartis entre diverses dates futures.

Le calcul des primes d'une opération d'assurance-vie s'appuie sur le principe d'équivalence: il doit y avoir équivalence entre les engagements de l'assureur et ceux de l'assuré.

Ceux-ci étant échelonnés dans le temps, ils doivent bien sûr préalablement être actualisés à une même date, conventionnellement à l'origine du contrat.

Le principe de base est donc:

valeur actuelle à l'origine des engagements de l'assureur = valeur actuelle à l'origine des engagements de l'assuré.

Deux outils interviennent dans le calcul:

- la table de mortalité, permettant de quantifier les probabilités de survenance des événements aléatoires sous-jacents au produit;

- le taux d'actualisation, aussi appelé taux d'intérêt technique, fixé par voie réglementaire.

En Belgique, l'arrêté royal du 15 juillet 1985 permet, depuis le $1^{\text {er }}$ janvier 1986, aux compagnies d'utiliser un taux compris entre $4 \%$ et $4,75 \%$. Précédemment, le taux était univoque (4\% entre 1969 et 1986$)$.

Actuellement, la majorité du secteur utilise un taux de $4,75 \%$, un des plus élevés d'Europe.

D'une manière générale, le taux d'intérêt technique s'est toujours situé entre $3 \%$ et $5 \%$, quels que soient le pays et l'époque.

\section{Le calcul des réserves}

Comme indiqué précédemment, une partie des primes versées par l'assuré est mise en réserve et capitalisée de manière à atteindre au terme du contrat la prestation prévue.

On peut donc définir un principe de calcul des réserves mathématiques, dit rétrospectif, sur la base de la formule:

réserve $=$ valeur capitalisée des primes versées

- valeur capitalisée du risque déjà couvert

La caractéristique principale d'un contrat d'assurance-vie est la suivante: le taux de capitalisation intervenant dans cette formule est, non pas le taux de rendement réalisé, mais le taux d'intérêt technique fixé à l'émission du contrat.

\section{Le taux technique}

L'assureur-vie utilise donc son taux technique, non seulement pour estimer a priori la prime, mais également pour calculer a posteriori la réserve d'épargne de l'assuré. 
Il s'agit d'une obligation de résultats et non d'une obligation de moyens.

Cette contrainte résulte précisément du fait que les engagements des deux parties sont fixées en francs à l'origine et sont ne varietur dans la vie du contrat. priori.

Bien sûr, les taux de rendement réellement réalisés diffèrent de ce taux technique a

Une correction a posteriori se fait grâce a un boni, appelé participation bénéficiaire, venant s'ajouter au contrat de base. Le taux de participation est fixé par chaque assureur.

L'assurance repose donc sur le schéma:

taux d'actualisation $=$ taux technique a priori $=$ taux de rendement minimal taux de rendement a posteriori $=$ taux technique + taux de participation

Ce taux de participation a parfois corrigé le taux technique dans des proportions très importantes (année 1981-1982: le taux de participation atteignait 6,5\% alors que le taux technique se montait à $4 \%$ ).

La fixation du taux technique pose un dilemme:

- ce taux ne peut être trop élevé, car il s'agit pour l'assureur d'un engagement ferme à long terme: un assuré souscrivant un contrat verra tous ses versements futurs fixés à l'origine du contrat, gratifiés d'un taux de rendement au moins égal au taux technique fixé à la conclusion du contrat;

- ce taux doit tenir compte des conditions financières du moment, de manière à offrir un produit compétitif par rapport à d'autres formes d'épargne, les tarifs étant construits sur ce seul taux technique.

Pour estimer ce taux (fixé rappelons-le par voie réglementaire), on pourrait se baser sur la relation de Fisher: le taux d'intérêt nominal est la somme d'un taux de rendement réel et d'une anticipation d'inflation.

Pour autant que l'on puisse défendre à long terme une stabilité de ce taux de rendement réel, le taux technique pourrait être donné par:

taux technique $=$ taux réel à long terme $+\lambda \times$ anticipation d'inflation avec $0 \leq \lambda<1$ $\lambda=0$ conduit à une politique particulièrement prudente où l'assureur ne s'engage que sur un rendement hors inflation supposé stable à long terme.

Dans un tel contexte, on pourrait à la limite ne presque jamais modifier le taux technique. Ce dernier pourrait, bien sûr, varier d'un pays à l'autre.

Cette politique conduit à ne pas tenir compte des fortes hausses des taux nominaux en période de forte inflation. 
Ce taux réel à long terme est généralement estimé entre 2 et $3 \%$. $0<\lambda<1$ consiste à tenir compte en partie d'une anticipation d'inflation jugée raisonnable à long terme.

Il faut noter en pratique que le taux technique n'est revu que rarement.

Toute modification du taux technique est, bien sûr, d'application pour les nouveaux contrats.

En Belgique, on l'applique aussi aux versements futurs des contrats existants, si cette opération est bénéfique à l'assuré (adaptation des portefeuilles).

Une telle pratique n'est pas toujours d'application dans d'autres pays, où coexistent alors à une même date différents tarifs, suivant la date de souscription du contrat.

Il convient enfin d'insister sur le fait que l'assureur travaille sur du long terme et doit à ce titre utiliser des moyennes à long terme de taux.

\section{Rentes à deux taux}

D’une manière générale, l'assurance vie est basée sur un taux d'intérêt technique unique, quels que soient la durée et le type d'opération envisagée.

Néanmoins, l'arrêté royal du 15 juillet 1985 a dérogé à ce principe, pour les opérations de rente viagère immédiate. Cette technique dite des rentes à deux taux consiste à utiliser deux taux d'actualisation: le premier, utilisé pendant une durée déterminée (par exemple 5 ans), est censé refléter des conditions de placement du moment en termes nominaux, tandis qu'au-delà de cette période, il est fait usage du taux d'intérêt technique classique.

On parvient ainsi à concilier la prudence à long terme et le réalisme à court terme.

\section{B. Financement collectif de régimes de retraite}

Des préoccupations liées au taux d'actualisation jouent également dans d'autres aspects que la nature même du taux technique.

Nous développons ci-dessous un exemple où les paramètres financiers occupent une place centrale en assurance-vie.

Un des problèmes importants d'estimation actuarielle consiste à planifier le financement d'un régime de retraite complémentaire souscrit par une entreprise pour son personnel ou pour certaines catégories de celui-ci.

Ces régimes ont pour but d'apporter un complément aux prestations légales issues de la sécurité sociale. La technique de l'assurance permet de fixer les primes à verser lors de la souscription d'un tel plan. 
Néanmoins, de manière à apprécier le coût d'un tel plan lors de son installation, il convient souvent d'effectuer la projection de la charge sur un certain horizon (par exemple 20 ans) de manière à tenir compte de deux types d'évolution:

- l'évolution démographique de l'entreprise assurée (vieillissement, remplacement, turn over)

- l'évolution financière (augmentation des traitements, capitalisation des réserves du régime, etc.).

Le taux d'intérêt technique étant le plus généralement supérieur à un taux de rendement réel hors inflation à long terme, on travaille non pas en francs constants, mais en francs courants.

Un certain nombre de paramètres interviennent alors dans la projection à effectuer:

- le taux d'inflation

- le taux de progression des salaires (inflation + barème + carrière) $T$

- le taux de rendement total

- le taux de participation bénéficiaire

\section{I}

$\mathrm{R}$

$\mathrm{PB}=\mathrm{R}-4,75 \%$

Un des buts de la projection est la détermination d'un taux de dotation qui, appliqué chaque année à la masse salariale de l'entreprise, dégage le versement total de l'année. charges.

Il existe diverses méthodes de financement qui permettent d'obtenir divers profils de

Une des méthodes les plus usitées, l'«Aggregate cost method», conduit à la formule:

taux de dotation $=\frac{\Sigma \text { primes projetées actualisées }}{\Sigma \text { masses salariales projetées actualisées }}$

L'actualisation se faisant au taux de rendement total $R$.

Plutôt que de verser des primes qui peuvent connaître des variations très importantes, l'entreprise verse donc une dotation stable en pourcentage de sa masse salariale.

Deux types de phénomènes interviennent dans la détermination de ce taux de charge:

- des éléments objectifs (importance des garanties choisies, composition du groupe)

- des éléments subjectifs (les paramètres de projection du régime).

En ce qui concerne ces paramètres, il n'existe pas de contraintes précises en Belgique.

Il est néanmoins conseillé d'utiliser des paramètres satisfaisant aux contraintes suivantes:

$0<\mathrm{R}-\mathrm{I}<3 \%$

$0<\mathrm{T}-\mathrm{I}<3 \%$

$\mathrm{R}>\mathrm{T}$ 
La première inégalité conduit à un taux de rendement réel positif, mais inférieur à $3 \%$.

La seconde inégalité suppose une progression des salaires supérieure à l'inflation (indexation), mais ne la dépassant pas de plus de $3 \%$.

La dernière inégalité stipule un taux de rendement supérieur au taux de croissance des salaires.

Montrons sur un exemple l'importance, non pas de la valeur absolue de ces paramètres, mais des différentiels.

Considérons pour cela un plan prévoyant pour une carrière complète de 40 ans le versement à partir de l'âge de la retraite d'une rente égale à $70 \%$ du dernier traitement annuel de la carrière, diminué de la pension légale.

Rente $=\frac{N}{40} \cdot\left(70 \% T^{\text {annuel }}-\mathrm{PL}\right)$

$(\mathrm{N}=$ durée de la carrière $)$

Pour une entreprise jeune d'âge homogène fixé à 25 ans, on obtient les taux suivants les scénarios envisagés: 
Premier groupe d'hypothèses:

\begin{tabular}{rrrrrrr} 
& T & I & R & $\begin{array}{r}\text { Intérêt } \\
\text { réel }\end{array}$ & Barème & $\begin{array}{r}\text { Taux de } \\
\text { dotation }\end{array}$ \\
\hline 1 & $5,50 \%$ & $3,50 \%$ & $6 \%$ & $2,5 \%$ & $2 \%$ & $7,71 \%$ \\
2 & $4,25 \%$ & $2,25 \%$ & $4,75 \%$ & $2,5 \%$ & $2 \%$ & $7,72 \%$ \\
3 & $7,50 \%$ & $5,50 \%$ & $8 \%$ & $2,5 \%$ & $2 \%$ & $7,69 \%$
\end{tabular}

Deuxième groupe d'hypothèses:

abaissement de 1 point du taux d'intérêt réel

\begin{tabular}{|c|c|c|c|c|c|c|}
\hline & $\mathrm{T}$ & I & $\mathrm{R}$ & $\begin{array}{r}\text { Intérêt } \\
\text { réel }\end{array}$ & Barème & $\begin{array}{l}\text { Taux de } \\
\text { dotation }\end{array}$ \\
\hline 1 & $5,50 \%$ & $3,50 \%$ & $5 \%$ & $1,5 \%$ & $2 \%$ & $9,31 \%$ \\
\hline 3 & $7,50 \%$ & $5,50 \%$ & $7 \%$ & $1,5 \%$ & $2 \%$ & $9,26 \%$ \\
\hline
\end{tabular}

Troisième groupe d'hypothèses:

taux d'intérêt réel fixé à $4,5 \%$

$\begin{array}{rrrrrr}\mathrm{T} & \mathrm{I} & \mathrm{R} & \begin{array}{r}\text { Intérêt } \\ \text { réel }\end{array} & \text { Barème } & \begin{array}{r}\text { Taux de } \\ \text { dotation }\end{array} \\ & & & & & \\ 0 \% & 3,50 \% & 8 \% & 4,5 \% & 2 \% & 5,12 \% \\ 0 \% & 5,50 \% & 10 \% & 4,5 \% & 2 \% & 5,15 \%\end{array}$

Quatrième groupe d'hypothèses:

exemple extrême: taux réel nul

\begin{tabular}{rrrrrr}
$\mathrm{T}$ & $\mathrm{I}$ & $\mathrm{R}$ & $\begin{array}{r}\text { Intérêt } \\
\text { réel }\end{array}$ & Barème & $\begin{array}{r}\text { Taux de } \\
\text { dotation }\end{array}$ \\
\hline $7,50 \%$ & $5,50 \%$ & $5,50 \%$ & $0 \%$ & $2 \%$ & $12,02 \%$
\end{tabular}

\section{Conclusion:}

Le taux de charge varie donc de plus du simple au double lorsque le taux d'intérêt réel passe de $0 \%$ à $4,5 \%$. mètres.

Par contre, ce taux est très stable lorsqu'on modifie de manière uniforme tous les para-

Le taux d'intérêt réel à long terme constitue donc le paramètre central dans l'estimation du coût d'un tel plan de prévoyance.

Annexe: Evolution des taux d'intérêt nominaux et réels de 1954 à 1984. 
Evaluation des taux réels moyens à long et à court termes

\begin{tabular}{|c|c|c|c|c|c|c|}
\hline Années & $\begin{array}{r}\text { Indice } \\
\text { des prix }\end{array}$ & $\begin{array}{r}\text { Taux } \\
\text { d'intérêt } \\
\text { nominal } \\
\text { à CT }\end{array}$ & $\begin{array}{r}\text { Taux } \\
\text { d'inflation } \\
\text { annuel }\end{array}$ & $\begin{array}{c}\text { Taux } \\
\text { d'intérêt } \\
\text { réel à CT non }\end{array}$ & $\begin{array}{r}\text { Taux } \\
\text { d'intérêt } \\
\text { ninal à LT }\end{array}$ & $\begin{array}{r}\text { Taux } \\
\text { d'intérêt } \\
\text { réel à LT }\end{array}$ \\
\hline 1954 & 1,0000 & 0,025 & 0,0176 & 0,0074 & 0,0478409 & 0,0246565 \\
\hline 1955 & 0,9950 & 0,025 & $-0,004972$ & 0,299716 & 0,0449882 & 0,0165316 \\
\hline 1956 & 1,0277 & 0,025 & 0,0328337 & $-0,007834$ & 0,0485849 & 0,0257365 \\
\hline 1957 & 1,0601 & 0,0271 & 0,0315595 & $-0,004460$ & 0,0534510 & 0,0303957 \\
\hline 1958 & 1,0739 & 0,0327 & 0,0129522 & 0,0197478 & 0,0513033 & 0,0281601 \\
\hline 1959 & 1,0871 & 0,0325 & 0,0123457 & 0,0201543 & 0,0512116 & 0,0245730 \\
\hline 1960 & 1,0904 & 0,0343 & 0,0030488 & 0,0312512 & 0,05 & 0,0248083 \\
\hline 1961 & 1,1011 & 0,0375 & 0,0097699 & 0,0277301 & 0,0569236 & 0,0250549 \\
\hline 1962 & 1,1167 & 0,0312 & 0,0141905 & 0,0170095 & 0,0534982 & 0,0182584 \\
\hline 1963 & 1,1406 & 0,0317 & 0,0214119 & 0,0102881 & 0,0570092 & 0,0150401 \\
\hline 1964 & 1,1882 & 0,0425 & 0,0417186 & 0,0007814 & 0,0625 & 0,0261763 \\
\hline 1965 & 1,2365 & 0,042 & 0,0406455 & 0,0013545 & 0,0636990 & 0,0279345 \\
\hline 1966 & 1,2881 & 0,448 & 0,0417385 & 0,0030615 & 0,0675 & 0,0290450 \\
\hline 1967 & 1,3248 & 0,0475 & 0,0284874 & 0,0190126 & 0,0675 & 0,0268985 \\
\hline 1968 & 1,3610 & 0,044 & 0,0273410 & 0,0166590 & 0,0660362 & 0,0071034 \\
\hline 1969 & 1,4122 & 0,0806 & 0,0375718 & 0,0430282 & 0,0810851 & 0,0088528 \\
\hline 1970 & 1,4673 & 0,0715 & 0,0390612 & 0,0324388 & 0,0812564 & 0,0011640 \\
\hline 1971 & 1,5310 & 0,0606 & 0,0434011 & 0,0171989 & 0,0825 & $-0,005591$ \\
\hline 1972 & 1,6143 & 0,0543 & 0,0544302 & $-0,000130$ & 0,0709308 & $-0,012484$ \\
\hline 1973 & 1,7266 & 0,0748 & 0,0695117 & 0,0052883 & 0,0771517 & $-0,006799$ \\
\hline 1974 & 1,9455 & 0,1156 & 0,1268340 & $-0,011234$ & 0,0814477 & 0,0041627 \\
\hline 1975 & 2,1941 & 0,0813 & 0,1277683 & $-0,046468$ & 0,1 & 0,0313798 \\
\hline 1976 & 2,3951 & 0,1034 & 0,0916055 & 0,0117945 & 0,1 & 0,0331074 \\
\hline 1977 & 2,5651 & 0,0841 & 0,0709697 & 0,0131303 & 0,0980102 & 0,0324336 \\
\hline 1978 & 2,6799 & 0,0798 & 0,0447623 & 0,0350377 & 0,085 & 0,0158905 \\
\hline 1979 & 2,7997 & 0,1025 & 0,0446996 & 0,0578004 & & \\
\hline 1980 & 2,9858 & 0,1398 & 0,0664637 & 0,0733363 & & \\
\hline 1981 & 3,2138 & 0,1511 & 0,0763559 & 0,0747441 & & \\
\hline 1982 & 3,4938 & 0,1438 & 0,0871455 & 0,0566545 & & \\
\hline 1983 & 3,7618 & 0,1118 & 0,0767042 & 0,0350958 & & \\
\hline 1984 & 4,0007 & 0,1162 & 0,0634991 & 0,0527009 & & \\
\hline
\end{tabular}

Taux moyen réel à court terme de 1954 à 1984: $\quad 0,0205783$ 